LAWRENCE LIVERMORE N A T IO N A L LABORATORY

Pulse requirements for electron diffraction imaging of single biological molecules

Stefan Hau-Riege, Richard London, Henry Chapman

October 28, 2004 
This document was prepared as an account of work sponsored by an agency of the United States Government. Neither the United States Government nor the University of California nor any of their employees, makes any warranty, express or implied, or assumes any legal liability or responsibility for the accuracy, completeness, or usefulness of any information, apparatus, product, or process disclosed, or represents that its use would not infringe privately owned rights. Reference herein to any specific commercial product, process, or service by trade name, trademark, manufacturer, or otherwise, does not necessarily constitute or imply its endorsement, recommendation, or favoring by the United States Government or the University of California. The views and opinions of authors expressed herein do not necessarily state or reflect those of the United States Government or the University of California, and shall not be used for advertising or product endorsement purposes.

This work was performed under the auspices of the U.S. Department of Energy by University of California, Lawrence Livermore National Laboratory under Contract W-7405-Eng-48. 


\title{
Pulse requirements for electron diffraction imaging of single biological molecules
}

\author{
Stefan P. Hau-Riege, Richard A. London, and Henry N. Chapman \\ Lawrence Livermore National Laboratory, Livermore, California 94539, USA
}

The pulse requirements for electron diffraction imaging of single biological molecules are calculated. We find that the electron fluence and pulse length requirements imposed by the damage limit and by the need to classify the diffraction patterns according to their angular orientation cannot be achieved with today's electron beam technology. A simple analytical model shows that the pulse requirements cannot be achieved due to beam broadening due to spacecharge effects.

Crystallography is widely used for determining the structure of biological molecules [1]. In x-ray and electron crystallography, the intensity of the diffraction pattern of a periodic assembly of molecules is measured, and the electron density is reconstructed using phase retrieval algorithms [2]. Structure modifications due to radiation damage are prevented by sharing the incoming radiation among a large number of coherently scattering molecules [3]. A major challenge is the fabrication of the required uniform and large crystals, and many important biological molecules have resisted attempts at crystallization completely.

In an alternative approach that does not require crystallization, two-dimensional diffraction patterns of single isolated molecules are recorded. In the simplest scenario, individual, identical molecules are exposed to pulses one-by-one in random, unknown orientation. Schemes to pre-align the molecules have been suggested but are experimentally formidable [4]. If the molecules are not pre-aligned, the relative orientations of these patterns are then determined, and from these, a three-dimensional diffraction pattern is constructed. Since the scattering strength of an isolated molecule is significantly lower than of a crystal of molecules, much larger fluences are required, and radiation damage becomes a concern. The damage limitation can potentially be overcome by using extremely short and intense pulses to capture each image before the damage can manifest itself [5]. Neutze et al. suggested the possibility of using short xray pulses for high-resolution diffraction imaging of single isolated biological molecules [6].

In addition to x-rays, electrons are a likely candidate for diffraction imaging since their interaction cross section with biological materials is $\sim 10^{3}$ times larger than for photons. Recent advances have made it possible to attain sub-picosecond electron pulses with an unprecedented electron number density $[7,8]$. It is the goal of this paper to determine the pulse requirements for electron diffraction imaging of single biological molecules and to explore if such pulse are achievable with today's electron beam technology.

In single particle diffraction imaging, the measured diffraction patterns will be very noisy due to counting statistics, so that the limiting step will be the ability to classify the diffraction patterns according to their orientation. For x-ray imaging, Huldt et al. [9] developed an analytical model that determines the minimum number of incident photons 
per unit area (photon fluence) for a given the particle size and desired resolution. The analysis is based on determining to a certain confidence level if two noisy diffraction images show the same view of the sample or two different views. Their model assumes there is no damage, so that the result is independent of the pulse duration. Here we extend the model to electron beam diffraction imaging. We use a screened Rutherford cross section to describe the coherent elastic scattering of the electrons off atoms, which is suitable for large energies $(>10 \mathrm{keV})$ and small-atomic-number elements $(\mathrm{Z}<30)$. The cross section in differential form is given by $[10,11]$

$$
\frac{\mathrm{d} \sigma}{\mathrm{d} \Omega}\left[\mathrm{A}^{2} / \text { atom }\right] \approx 5.21 \times 10^{-5} \frac{\mathrm{Z}^{2}}{\mathrm{E}^{2}}\left(\frac{\mathrm{E}+511}{\mathrm{E}+1024}\right)^{2} \frac{1}{\left(\sin ^{2}\left(\frac{\phi}{2}\right)+\alpha\right)^{2}},
$$

where $\mathrm{E}$ is the electron energy in $\mathrm{keV}, \phi$ is the scattering angle, $\mathrm{Z}$ is the atomic number, and $\alpha$ is the screening factor that can be estimated by [12]

$$
\alpha \approx 3.4 \times 10^{-3} \frac{Z^{0.67}}{E} .
$$

Figure 1 shows the electron fluence requirements to classify diffraction images with $90 \%$ confidence at $10 \mathrm{keV}, 100 \mathrm{keV}$, and $1 \mathrm{MeV}$, as a function of particle size and image resolution. We assumed the chemical stoichiometry of the anthrax lethal factor $\left(\mathrm{H}_{51.61} \mathrm{C}_{30.77} \mathrm{~N}_{8.16} \mathrm{O}_{9.40} \mathrm{~S}_{0.60}\right)$ [13], a particular protein of current interest, and a mass density of $1.35 \mathrm{~g} / \mathrm{cm}^{3}$. Other protein molecules have quite similar composition and density. Figure 1 shows that reducing the molecule size or improving the resolution requires higher fluence. Also, larger fluences are required when higher energy electrons are used. This is primarily due to a decrease of the solid angle $\Omega$ spanned by each pixel at higher energy. If we assume that all pixels are independent samples of the molecular transform according to the sampling theorem [14], then the distance between independent pixels in reciprocal space is $1 / 2 \mathrm{a}$, where a is the molecule radius, and [9]

$$
\Omega=\left(\frac{\lambda}{2 a}\right)^{2}
$$

$\lambda$ is the de Broglie wavelength of the electrons. Even though the total elastic scattering cross section decreases at higher electron energies, the differential scattering cross section actually increases at small angles according to Equation (1). For instance, when the electron energy is increased from $100 \mathrm{keV}$ to $1 \mathrm{MeV}$, the differential elastic scattering cross section at the resolution to which the images are classified increases 2.4 times, the de Broglie wavelength decreases 4.2 times, and we find that the required fluences increase approximately 7 fold. This estimate is consistent with the results shown in Figure 1. 
Just as with $\mathrm{x}$ rays, electron irradiation causes energy transfer to the molecule and subsequent damage. At $30 \mathrm{keV}$, for instance, for every elastically scattered electron about two primary electrons damage the biological molecule through impact ionization [15]. The nature of the damage is similar to the case of $x$-ray irradiation $[6,16,17]$. The secondary electrons escape the molecule initially and charge it up positively. Since the energy of the secondary electrons is only of the order of $25 \mathrm{eV}$ [18], the molecule charge quickly becomes large enough to trap the secondary electrons electrostatically. The trapped electrons collide with each other frequently $(\sim 1 / \mathrm{fs})$ and their energy distribution becomes Maxwellian after a few collisions. They then relax in position to form a neutralizing cloud around the positively charged ions. The particle then assumes a roughly two-zone structure, consisting of a neutral core and a thin positively charged outer shell, which extends to the boundary of the particle. On a longer timescale of order $10 \mathrm{fs}$, the repulsive electrostatic forces between the ions cause a macroscopic motion of the whole molecule, called a Coulomb explosion. The trapped electrons damage the molecule further through electron impact ionization.

We previously developed a hydrodynamic continuum model for the physics that leads to damage when a small particle absorbs a large x-ray dose [17]. This model has been modified to treat the electron irradiation case. It includes ionization processes and Coulomb-force driven atomic motion. Further, trapping of electrons, Debye shielding, non-uniform collisional ionization, and three-body recombination effects are all included in the model. The molecule is divided into equidistant radial zones, i.e. spherical shells. We track the zone boundaries as the molecule expands. Figure 2 (a) shows the outward motion of selected spatial zones of a molecule of radius $150 \mathrm{~A}$, irradiated by an electron pulse of flux $5 \times 10^{7} \mathrm{ph} / \mathrm{fs} /(100 \mathrm{~nm})^{2}$ for $100 \mathrm{fs}$. Only the charged outer layers of the molecule expand, whereas the neutral molecule core does not move with time. Figure 2 (b) shows the ionization of carbon, the dominant interacting constituent of biological molecules. Initially, the number of atoms in state $(2,3)$ increases with time due to (primary) electron impact ionization of neutral atoms, state $(2,4)$. The atoms then continue to be ionized through primary and to a lesser extent through secondary ionization of the valence electrons by the beam electrons and trapped electrons, respectively. Figure 2 (c) shows the time evolution of the average electron energy. The average electron temperature increases with time due to the increasingly higher initial energy of the secondary electrons with increasing ionization states of the ions [18] and the eventual reduction of the number of electrons due to three-body recombination. We further found that the shorter the pulse the less is the amount of damage by the trapped secondary electrons and also the shorter is the time for the Coulomb-driven motion of the atoms in the outer part of the molecule.

In order to assess the effect of the damage dynamics on the quality of the diffraction image, we calculated the time-integrated diffraction pattern of a pseudo molecule, and compared it with the diffraction pattern of a hypothetically undamaged sample. The pseudo molecule was generated by placing atoms randomly within a spherical volume according to the stoichiometry and mass density. The motion and ionization are 
calculated according to the continuum model described above. We follow the treatment by Neutze et al [6], and define a measure R for the effect of damage on the image quality,

$$
R=\sum_{\mathbf{u}}\left|\frac{K^{-1} \sqrt{I_{\text {real }}(\mathbf{u}, \Omega)}-\sqrt{I_{\text {ideal }}(\mathbf{u}, \Omega)}}{\sum_{\mathbf{u}^{\prime}} \sqrt{I_{\text {ideal }}\left(\mathbf{u}^{\prime}, \Omega\right)}}\right|,
$$

with

$$
K=\frac{\sum_{\mathbf{u}} \sqrt{I_{\text {real }}(\mathbf{u}, \Omega)}}{\sum_{\mathbf{u}} \sqrt{I_{\text {ideal }}(\mathbf{u}, \Omega)}} .
$$

$\mathrm{I}(\mathbf{u}, \Omega)$ is the mean number of elastically scattered photons to be detected by an idealized detector pixel of projected solid angle $\Omega$ centered at a positional vector, $\mathbf{u}$. $\mathrm{I}_{\text {real }}$ corresponds to the damaged molecule, and $\mathrm{I}_{\mathrm{ideal}}$ corresponds to the fictitious undamaged molecule. The degradation factor, R, strongly depends on the resolution up to which the ideal and the real images are compared, as shown in Figure 3. For given irradiation conditions, the R-factor increases as we demand higher resolution. We also found that the $\mathrm{R}$ factor is strongly affected by the beam parameters. As also shown in Figure 3, lower fluences or shorter electron pulses lead to lower R factors, and therefore higher-quality diffraction images, since the molecule is less damaged. If the damage of the molecule is too severe, the diffraction image is insufficient to allow image reconstruction. This poses an upper limit, $\mathrm{R}_{\max }$, on the $\mathrm{R}$ factor. $\mathrm{R}_{\max }$ can be used to determine the maximum achievable resolution from a damage point of view, and a value of $20 \%$ has proven to be sufficient [1].

We have combined the results from the damage simulations with the results from the theory for the effect of counting statistics on image resolution (classification) to obtain the maximum electron pulse length. Overlaid in Figure 1 are the electron pulse lengths as a function of image resolution, particle size, and electron fluence. For example, to achieve 4 A resolution for a molecule of radius $100 \mathrm{~A}$ with $100 \mathrm{keV}$ electrons, a fluence of $9 \times 10^{6}$ electrons in a $100 \mathrm{~nm}$ spot and a bunch length of $2 \mathrm{ps}$ or shorter are required. For comparison, a fluence of $2 \times 10^{12}$ photons in a $100 \mathrm{~nm}$ spot and a pulse length of $2 \mathrm{fs}$ are required when a $12 \mathrm{keV}$ x-ray pulse is used instead [19]. For molecules with radii between $50 \mathrm{~A}$ and $200 \mathrm{~A}$, generally pulse lengths of the order of 0.5 to $10 \mathrm{ps}$ are required.

Our calculations show that the pulse length and fluence requirements electron diffraction imaging of single biological molecules with the requirement to classify the diffraction pattern according to their respective orientation are extremely demanding and can hardly be met with today's electron beam technology. A state-of-the-art ultra-fast high-intensity electron beam system was recently discussed by Siwick et al. [7]. They were able to produce a $30 \mathrm{keV}$ electron of $0.6 \mathrm{ps}$ length with 6000 electrons per pulse. The pulse length is in the right regime for biomolecular diffraction imaging. A pulse diameter of $100 \mu \mathrm{m}$ results in a fluence of approximately $10^{-1}$ electrons in a $100 \mathrm{~nm}$ spot. This 
fluence is more about a factor of $10^{8}$ too small. Similarly, Cao et al. [8] reported on an electron beam system that produces a $60 \mathrm{keV}$ electron beam of 0.3-2.5 ps length with 2000 to 6000 electrons per pulse, which also is not sufficient.

The very large electron beam intensities that are required for single particle diffraction imaging are not achievable due to limitations caused by space charge effects, i.e. the selfdispersion due to the Coulomb repulsion between electrons in the pulse, which is most significant when the electron density is high. The space charge effect leads to a strong dependence of electron pulse length on the beam intensity due to longitudinal pulse broadening [8]. Space charge broadening also occurs transversally, and in the Appendix we present a simple model to estimate the extent of this effect. If we assume $K_{0}=9 \times 10^{6}$ electrons in a $100 \mathrm{~nm}$ spot and a pulse length of $2 \mathrm{ps}$, then, using Equation (11) of the Appendix, $\mathrm{k}=3.6 \times 10^{-4}$ for $100 \mathrm{keV}$ electrons. Using Equation (12) of the Appendix, we obtain an increase of a factor of 5 in beam diameter over a drift distance of $10 \mu \mathrm{m}$, and a factor of 1310 in beam diameter over a drift distance of $1 \mathrm{~mm}$, which shows that an electron beam of that fluence will quickly diverge due to space-charge effects. Note that in this regime the beam diverges so fast that our assumption that the radius varies slowly over $\mathrm{z}$ is not valid anymore.

Our calculations show that with electron pulses, lower fluences are sufficient for diffraction imaging of biological molecules without pre-alignment than with x-rays, but these fluences are still very difficult to obtain and cannot be realized with today's technology. Our calculations also show that the pulse length requirements are extremely challenging. A very simple analytical estimate shows that an electron beam of the required fluence and pulse length for atomic resolution imaging would transversally broaden over a very short distance, thereby reducing the fluence to a level were image orientation cannot be performed reliably. To realize single molecule diffraction imaging using electrons the fluence requirement will need to be relaxed, for example by using prealignment schemes that orient the molecule in the laser beam [4]. If the molecules are pre-aligned, lower fluences below the damage threshold [3] can be used.

We thank Abraham Szoke and Henry Chapman for useful discussions. This work was performed under the auspices of the US Department of Energy by the University of California, Lawrence Livermore National Laboratory under Contract No. W-7405-Eng48. 


\section{References}

[1] H.M. Berman, J. Westbrook, Z. Feng, G. Gilliland, T.N. Bhat, H. Weissig, I.N. Shindyalov, P.E. Bourne, Nucl. Acids Res. 28235 (2000).

[2] R. Gerchberg, and W. Saxton, Optik 35, 237 (1972).

[3] R. Henderson, Quart. Rev. Biophys. 28, 2 (1995).

[4] J.C.H. Spende and R.B. Doak, Phys. Rev. Lett. 92, 198102 (2004).

[5] C. Solem and G.C. Baldwin, Science 218, 229 (1982).

[6] R. Neutze, W. Wouts, D. van der Spoel, E. Weckert, and J. Hajdu, Nature 406, 752 (2000).

[7] B.J. Siwick, J.R.Dwyer, R.E.Jordan, and R.J.D. Miller, Science 302, 1382 (2003).

B.J. Siwick, J.R.Dwyer, R.E.Jordan, and R.J.D. Miller, Chem. Phys. 299, 285 (2004).

[8] J. Cao, Z. Hao, H. Park, C. Tao, D. Kau, and L. Blaszczyk, Appl. Phys. Lett. 83, 1044 (2003).

[9] G. Huldt, A. Szoke, and J. Hajdu, J. Struct. Biol. 144, 219 (2003).

[10] D.E. Newbury and R.L. Myklebust, in Analytical Electron Microscopy 1981, ed. R.H. Geiss, p. 91, San Francisco Press, San Francisco, CA (1981).

[11] L. Reimer and E.R. Krefting, in Use of Monte Carlo Calculations in Electron Probe Microanalysis and Scanning Electron Microscopy, ed. K.F.L. Heinrich, D.E. Newbury, and H. Yakowitz, p. 45, U.S. Dept. of Commerce/National Bureau of Standards, NBS Special Publication \#460 (1976).

[12] H.E. Bishop, in Use of Monte Carlo Simulation in Electron Probe Microanalysis and Scanning Electron Microscopy, ed. K.F.L. Heinrich, D.E. Newbury, and H. Yakowitz, p. 5, U.S. Dept. of Commerce/National Bureau of Standards, NBS Special Publication \#460 (1976).

[13] H.M. Berman, J. Westbrook, Z. Feng, G. Gilliland, T.N. Bhat, H. Weissig, I.N. Shindyalov, P.E. Bourne, Nucl. Acids Res. 28, 235 (2000).

[14] E. T. Whittaker, Proc. Roy. Soc. Edinburgh, sec. A 35, 181 (1915). V. Kotel'nikov, Izd. Red. Upr. Svyazi RKKA, Moscow, Russian (1933). C. E. Shannon, Bell System Tech. J. 27, 379 (1948).

[15] M.A. Lennon, K.L. Bell, H.B. Gilbody, J.G. Hughes, A.E. Kingston, M.J. Murray, and F.J. Smith, J. Phys. Chem. Ref. Data 17, 1285 (1988). K.L. Bell, H.B. Gilbody, J.G. Hughes, A.E. Kingston, and F.J. Smith, J. Phys. Chem. Ref. Data 12, 891 (1983).

[16] Z. Jurek, G. Faigel, and M. Tegze, Europ. Phys. J. D 29, 217 (2004)

[17] S.P. Hau-Riege, R.A. London, and A. Szoke, Phys. Rev. E 69, 051906 (2004).

[18] Y.-K. Kim and M. E. Rudd, Phys. Rev. A 50, 3954 (1983).

[19] S.P. Hau-Riege and R.A. London, unpublished research. 


\section{Appendix}

Using the uniform beam model, we estimate the transverse broadening of an initially parallel and circular beam of radius $\mathrm{a}_{0}$ when it drifts over a distance $\mathrm{L}$ in the $\mathrm{z}$ direction. We assume that the beam length is much longer than the diameter, so that end effects are negligible, and we ignore longitudinal space charge effects. We assume that the beam radius $\mathrm{a}(\mathrm{z})$ varies with distance $\mathrm{z}$ slow enough so that the axial electric field and the radial magnetic field can be neglected. The charge density $\rho(z)$ is assumed to be uniform across each cross section of the beam. The electric current is constant along the beam and is given by

$$
\mathrm{I}=\frac{\mathrm{Q}}{\tau}=-\frac{\mathrm{eK}_{0} \pi \mathrm{a}_{0}^{2}}{\tau}
$$

where $K_{0}$ is the fluence of the electron pulse, $\tau$ the pulse duration, and e the fundamental charge. The radial electric field and the transversal magnetic field, respectively, are given by

$$
\begin{aligned}
& \mathrm{E}_{\mathrm{r}}(\mathrm{r}, \mathrm{z})=\frac{\mathrm{I}}{\pi \varepsilon_{0} \mathrm{v}_{0}} \frac{\mathrm{r}}{2 \mathrm{a}(\mathrm{z})^{2}} \text { and } \\
& \mathrm{B}_{\mathrm{t}}(\mathrm{r}, \mathrm{z})=\frac{\mu_{0} \mathrm{I}}{\pi} \frac{\mathrm{r}}{2 \mathrm{a}(\mathrm{z})^{2}},
\end{aligned}
$$

where $\mathrm{v}_{0}$ is the constant velocity of the electron bunch in the $\mathrm{z}$ direction. The radial force on a single electron is then

$$
\mathrm{F}_{\mathrm{r}}(\mathrm{r}, \mathrm{z})=-\mathrm{e}\left(\mathrm{E}_{\mathrm{r}}-\mathrm{v}_{0} \mathrm{~B}_{\mathrm{t}}\right)=-\frac{\mathrm{eE} \mathrm{r}(\mathrm{r}, \mathrm{z})}{\gamma^{2}}=\mathrm{m}_{\mathrm{e}} \frac{\mathrm{d}^{2} \mathrm{r}}{\mathrm{dt}^{2}}=\mathrm{m}_{\mathrm{e}} \mathrm{v}_{0}^{2} \frac{\mathrm{d}^{2} \mathrm{r}}{\mathrm{dz^{2 }}},
$$

with $\beta=v_{0} / c, \gamma=\left(1-\beta^{2}\right)^{-1 / 2}$, and $m_{e}$ is the electron mass. At the edge of the beam,

$$
\begin{aligned}
& \frac{\mathrm{d}^{2} \mathrm{a}(\mathrm{z})}{\mathrm{dz}}=\left(\frac{-\mathrm{eI}}{2 \pi \varepsilon_{0} \mathrm{~m}_{\mathrm{e}} \mathrm{v}_{0}^{3} \gamma^{2}}\right) \frac{1}{\mathrm{a}(\mathrm{z})} \equiv \mathrm{k} \frac{1}{\mathrm{a}(\mathrm{z})}, \text { with } \\
& \mathrm{k} \equiv \frac{\mathrm{e}^{2} \mathrm{~K}_{0} \mathrm{a}_{0}^{2}}{2 \varepsilon_{0} \mathrm{~m}_{\mathrm{e}} \tau \beta^{3} \mathrm{c}^{3} \gamma^{2}} .
\end{aligned}
$$

Figure 4 (a) shows the dependence of $k$ on the electron energy. The solution to this ordinary differential equation with $\mathrm{a}(0)=\mathrm{a}_{0}$ and $\mathrm{da}(0) / \mathrm{dz}=0$ is 


$$
a(z)=a_{0} \exp \left[\left(\operatorname{erfi}^{-1}\left[\frac{\mathrm{z}}{\mathrm{a}_{0}} \sqrt{\frac{2 \mathrm{k}}{\pi}}\right]\right)^{2}\right]
$$

where erfi is the imaginary error function. The beam radius a as a function of the drift length is shown in Figure 4 (b) for different values of $\mathrm{k}$. 


\section{Figure Captions}

Figure 1: Classification-limited resolution as a function of particle size and electron fluence for (a) $10 \mathrm{keV}$, (b) $100 \mathrm{keV}$, and (c) $1 \mathrm{MeV}$ electrons (solid lines). The curves are labeled with the electron fluence in units of number of photons in a $100 \mathrm{~nm}$ spot. The dashed lines show the damage-limited upper bound for the pulse lengths.

Figure 2: Damage caused by electron irradiation. (a) Motion of the atomic shells. Every third boundary is shown. (b) Ionization of carbon. (i,j) denotes a state with $\mathrm{i}$ electrons in the $\mathrm{K}$ shell and $\mathrm{j}$ electrons in the $\mathrm{L}$ shell. Only the states that reach densities of at least $5 \%$ of the initial density of the neutral state $(2,4)$ are shown. (c) Time evolution of the average electrons temperature, $\mathrm{T}_{\mathrm{e}}$. The molecule is of radius $150 \AA$ and is illuminated by an electron pulse of flux $5 \times 10^{7} \mathrm{ph} / \mathrm{fs} /(100 \mathrm{~nm})^{2}$ for $100 \mathrm{fs}$.

Figure 3: Degradation factor, $R$, as a function of resolution for different beam parameters. The graphs are labeled with the number of electrons per $100 \mathrm{~nm}$ diameter and the pulse length. The radius of the molecule is $150 \mathrm{~A}$.

Figure 4: (a) Dependence of the parameter k in Equation (9) on electron energy. (b) Dependence of beam radius on drift length for different values of $\mathrm{k}$. Not that for $\mathrm{K}_{0}=$ $9 \times 10^{6}$ electrons in a $100 \mathrm{~nm}$ spot and a pulse length of $500 \mathrm{fs}$, then $\mathrm{k}=1.44 \times 10^{-3}$ for 100 $\mathrm{keV}$ electrons. 


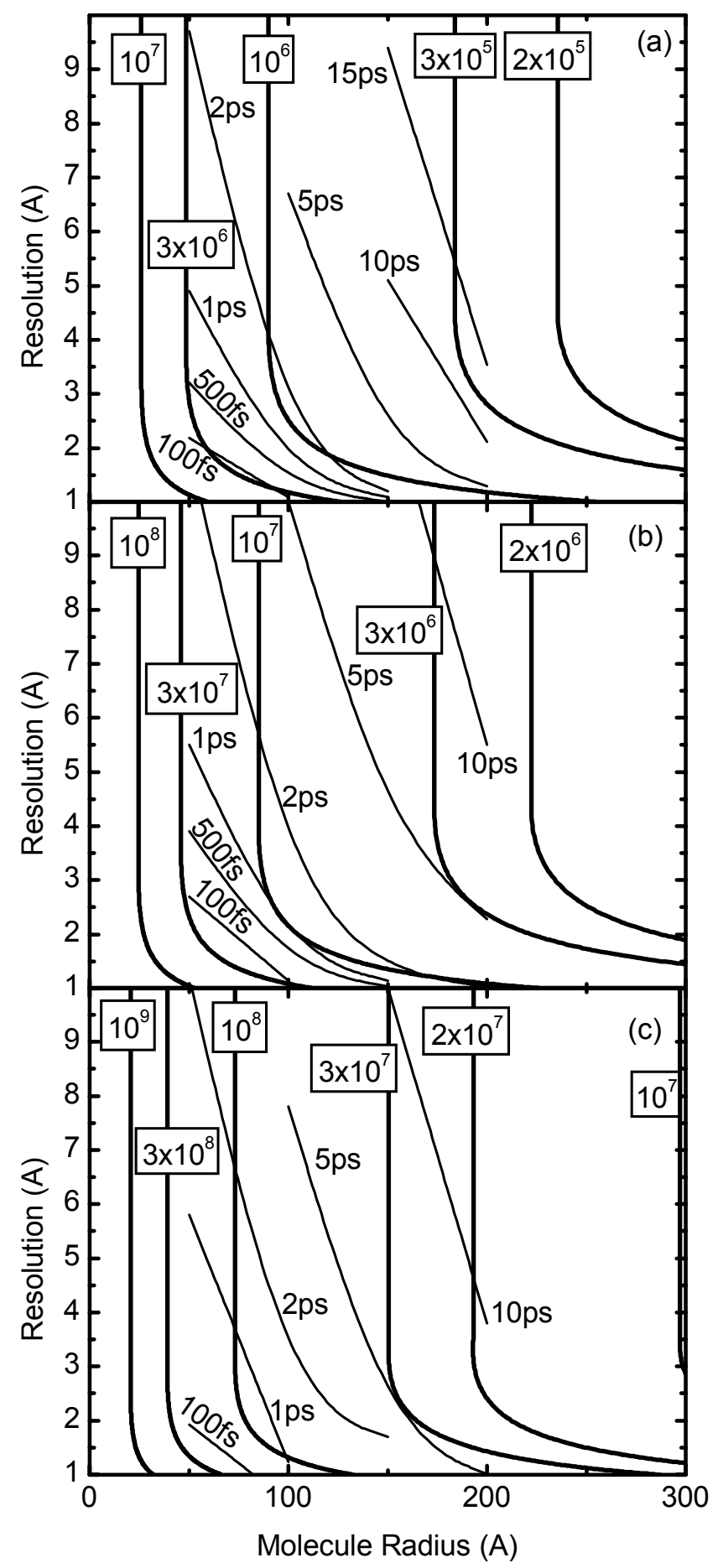

Figure 1 


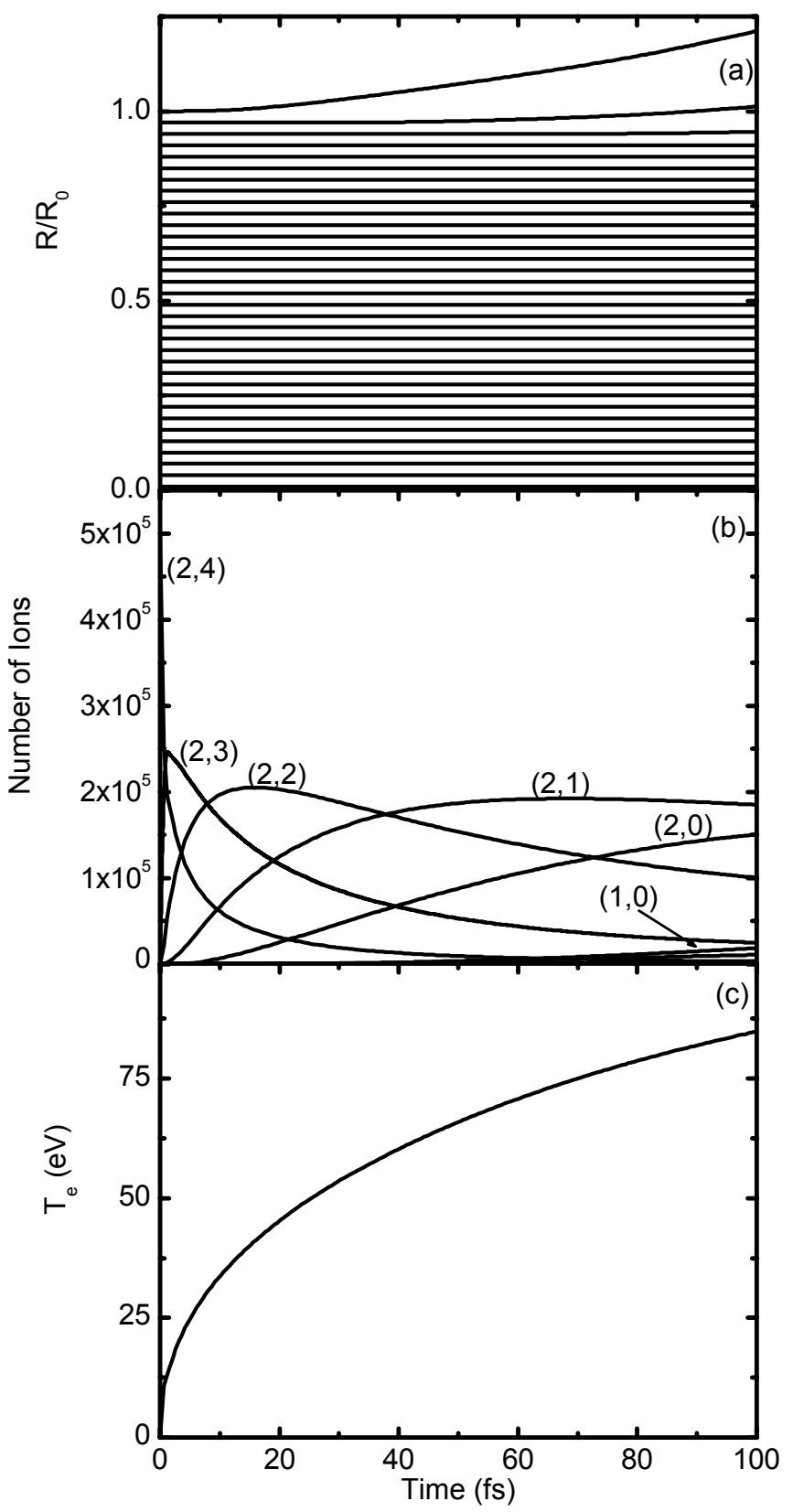

Figure 2 


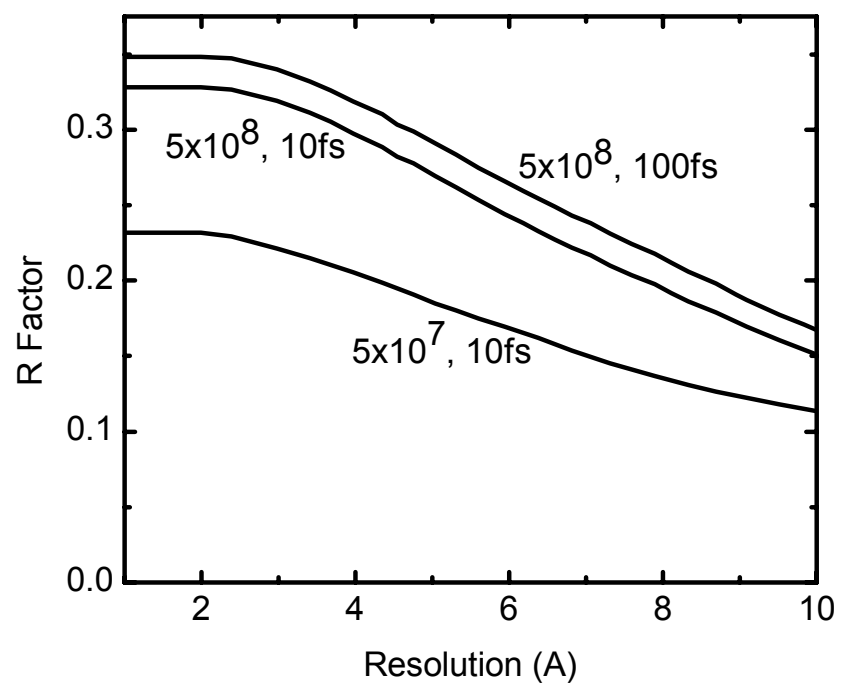

Figure 3 


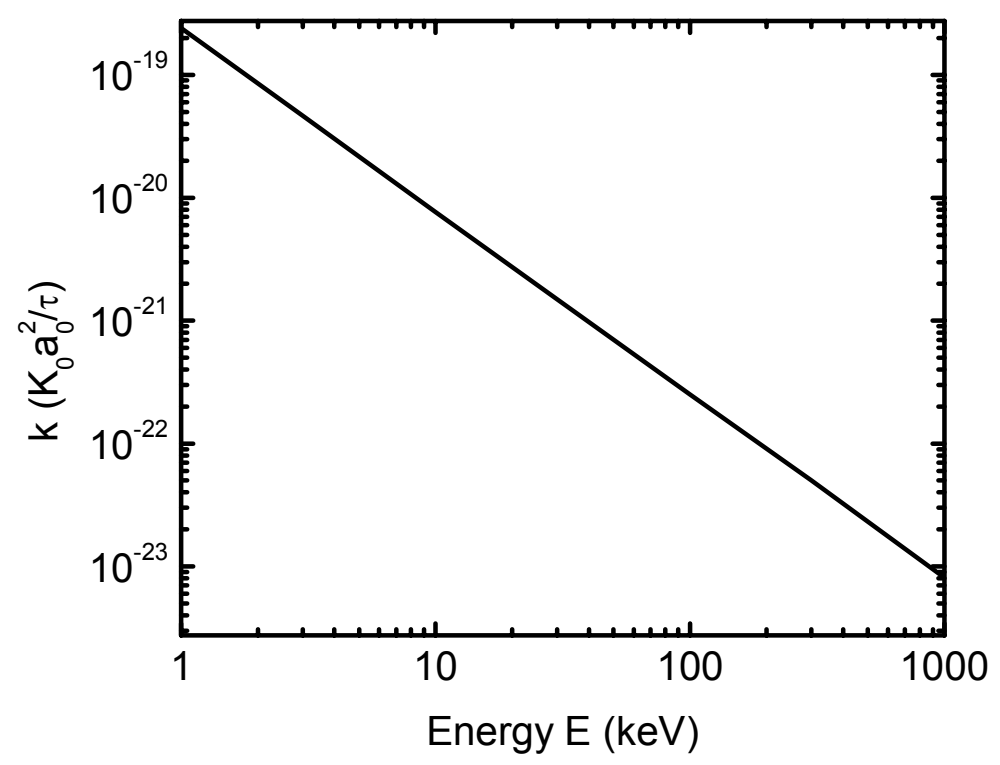

Figure 4 (a)

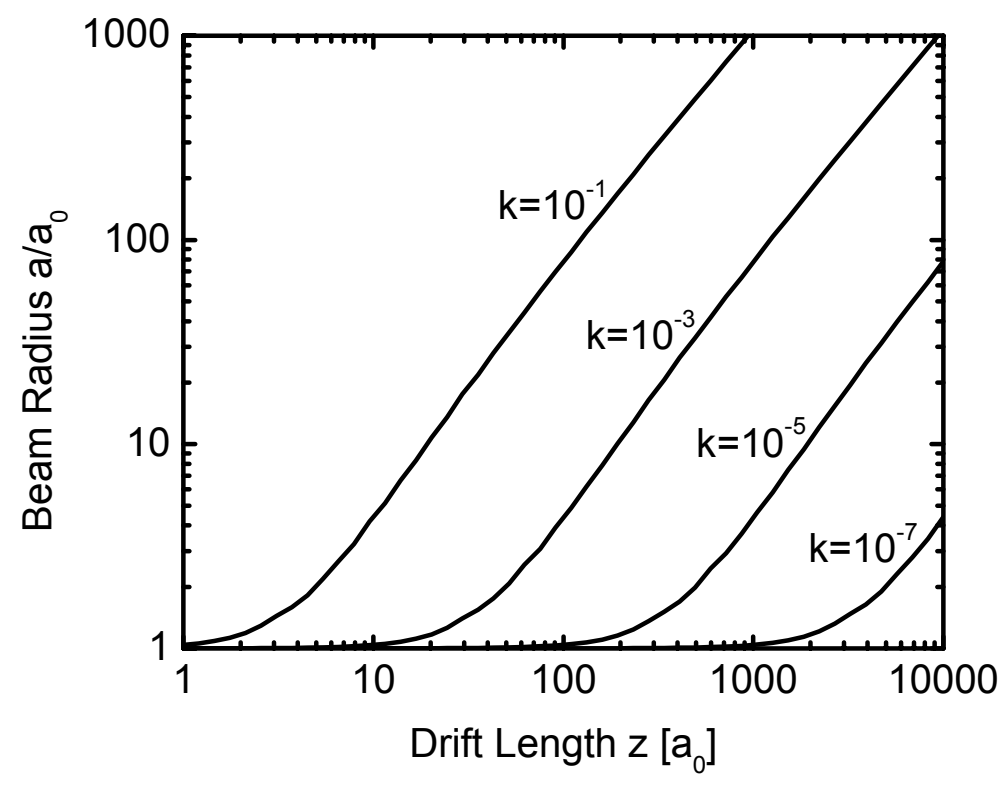

Figure 4 (b) 